\title{
Studies on readout channel thresholds of resistive MicroMegas for ATLAS Muon Spectrometer
}

\author{
M.T. Camerlingo ${ }^{a, b, *}$ and on behalf of the ATLAS Collaboration \\ ${ }^{a}$ University of Roma Tre, INFN Roma Tre, \\ Via della Vasca Navale 84, Rome, Italy \\ ${ }^{b} C E R N$, \\ Esplanade des Particules 1 - 1217, Geneve, Switzerland \\ E-mail: maria.teresa.camerlingo@cern.ch
}

On MicroMegas detectors for ATLAS Muon Spectrometer upgrade, tests on the noise are performed together with measurements of tracking efficiency, exploiting the cosmic rays as incoming particles. The MicroMegas readout channels are floating copper strips with different lengths, from $284.0 \mathrm{~mm}$ to $1990.0 \mathrm{~mm}$, capacitively coupled with carbon strips at few hundreds volts. Due to the wide strip length range, the strip capacitance affects the noise with different magnitude, leading a larger spread in the baseline along a tracking plane, respect to a configuration with same size strips. It also impacts the thresholds that are proportional to the baseline rms, with a settable factor. Especially if the variation in thresholds is large among close strips on a tracking layer, it compromises the time measurements for the $\mu$ TPC procedure. To uniform the thresholds on the plane, a singlechannel level correction can be applied, by an implemented trimmer in the electronic boards (based on VMM ASIC). Therefore, studies on the baseline and threshold were performed as a function of the strip length. After them, studies on the tracking efficiency were carried on as a function of the thresholds.

The Ninth Annual Conference on Large Hadron Collider Physics - LHCP2021

7-12 June 2021

Online

\footnotetext{
${ }^{*}$ Speaker
} 


\section{ATLAS resistive strip Micromegas design}

ATLAS [1] New Small Wheels upgrade project plans to replace the inner parts of the end-caps of the ATLAS Muon Spectrometer with new detector structures equipped by a combination of small-strip Thin Gap Chambers (sTGC) and resistive MicroMegas (MM) detectors [2] (see Figure 1). ATLAS MM detectors [2] are based on the resistive strip Micromegas proportional mode micro-pattern gaseous detectors [3] [4]. They will be mainly exploited as muon precision trackers, and as trigger detectors in occurrence. In the inner part of ATLAS Muon Spectrometer end-cap, a sector has a wedge shape and 8 tracking layers with planar geometry. To optimize the area coverage, large and small sectors were realized. For both sizes, the readout plane is segmented into 8192 floating copper strips with different lengths. For a large sector, as the one presented in the next section, the strip length varies from $426.7 \mathrm{~mm}$ to $1990 \mathrm{~mm}$. The readout strips are capacitively coupled with the carbon strips of the resistive spark suppression layer in Figure 2, read by VMM ASIC FE electronics [2]. If the size is large, the strip width and pitch are constant in the entire plane, they are $0.3 \mathrm{~mm}$ and $0.445 \mathrm{~mm}$, respectively. The nominal linear capacitance $(\mathrm{pF} / \mathrm{mm})$ is constant along the plane and, so, the capacitance linearly depends on the strip length in the first approximation of the strip as ideal parallel capacitor. The noise-dedicated studies in the next section were carried on Micromegas A07 sector, one of the $16 \mathrm{MM}$ sectors before the integration with sTGC modules and their mounting on the mechanical structure.

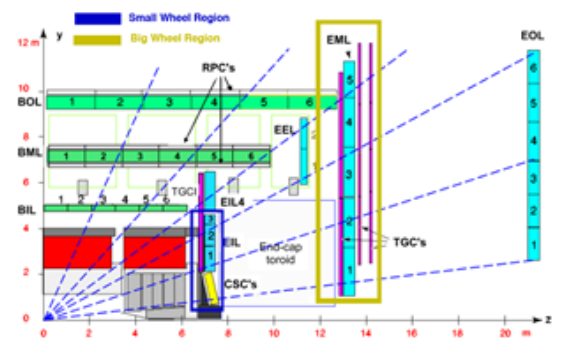

Figure 1: A quarter z-y view of ATLAS apparatus. In evidence, the three stations of the Muon spectrometer in the forward region: the End-cap Inner Large (EIL) End-cap Middle Large (EML) and End-cap Outer Large (EOL). The detector regions of the Small Wheel, that will be replaced by the new detectors, in dark blue box and Big Wheel in yellow box are also outlined [2].

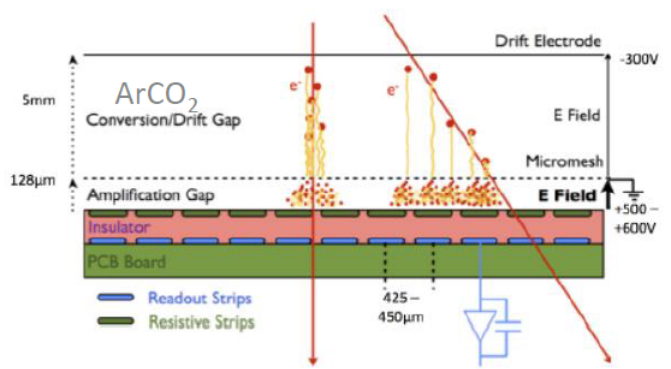

Figure 2: Sketch a single resistive Micromegas layer (Not in scale). 


\section{Noise dedicated studies on A07 Micromegas sector}

A Micromegas sector provides enough readout channels to perform statistical studies. The threshold of a readout channel is evaluated at the VMM-level (group of 64 consecutive strips). According to the Equation 1, its definition is built on the median (median baseline $_{\text {) }}$ and the rms $\left(\mathrm{RMS}_{\text {baseline }}\right)$ of the VMM-level baseline distribution. Depending on the strip length, the $\mathrm{RMS}_{\text {baseline }}$ varies from $\sim 1000 \mathrm{ENC}$ to $\sim 3000 \mathrm{ENC}$ for MM A07, and it is multiplied by $\mathrm{N}$, a settable parameter. To uniform the effective threshold, defined as the difference between the threshold and the baseline, a trimmer can decrease the single channel threshold in a $[0,30] \mathrm{mV}$ range reducing the threshold spread, as visible in Figure 3.

$$
\text { threshold }_{V M M}=\mathrm{N} \times \mathrm{RMS}_{\text {baseline }}+\text { median }_{\text {baseline }}
$$

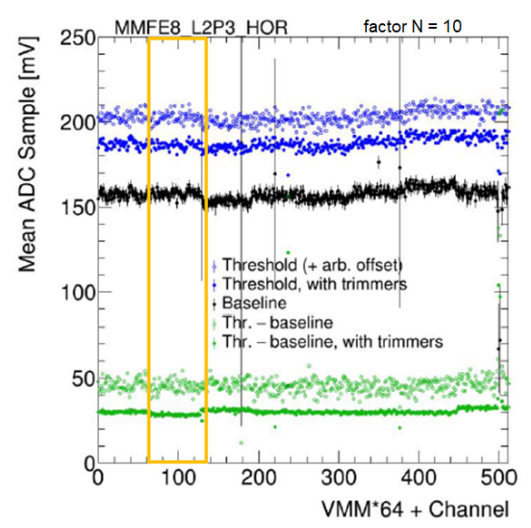

Figure 3: Example of baseline (black), threshold (blue) and effective threshold (green) as a function of readout channel indices of a group of $8 \mathrm{VMM}$, mounted on a same front-end card (MMFE8). The empty(full) markers of the thresholds are not corrected (are corrected) by the trimmer.

During the study on Micromegas A07 sector before its integration on the wheel, the $\mathrm{N}$ factor in Equation 1 was varied among $\{8,9,10,11,12,15\}$ with a fixed the VMM gain (equal to $9 \mathrm{mV} / \mathrm{fC}$ ), to investigate the detector performances when a larger threshold is applied respect to the actual nominal factor 9 . If the performances are still acceptable when tighter thresholds are applied, a higher $\mathrm{N}$ factor can be set to more efficiently suppress possible noise variations after the integration of the MM sector together with the STGC on the mechanical wheel support or after switching on the full wheel. Between the factor 9 and 12, the average effective threshold increases by $\sim 10 \mathrm{mV}$ (see Figure 4-Left). The average strip occupancy confirmed that factor 9 is the minimum applicable factor to have an acceptable uniformity of the occupancy on the plane. Indeed, the factor 8 can not suppress the noise on the longest strips (in Figure 4-Right). From the factor 15, a loss of events on the shortest strips is visible in Figure 4-Right.

\section{Tracking efficiency as a function of the effective threshold}

During this study, the cosmic rays were used as radiation source and a system of scintillators as external trigger system. The study was performed with the HV sectors at 

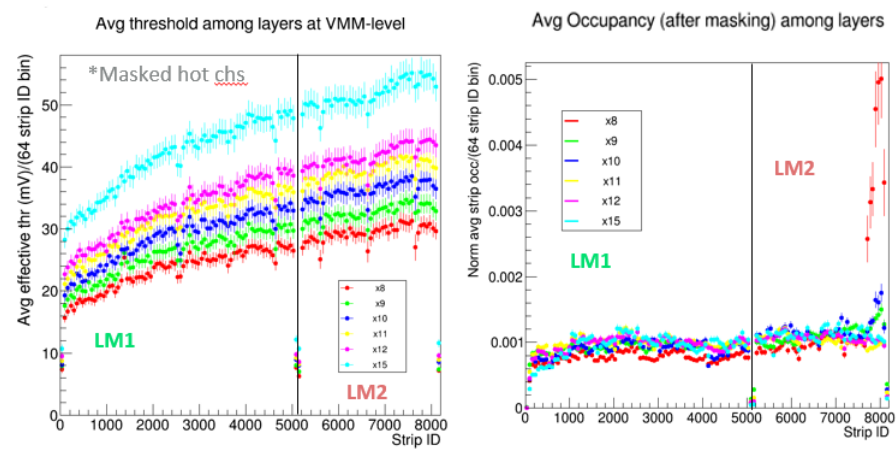

Figure 4: Average threshold at VMM-level on the A07 layers for $\mathrm{N}=\{8,9,10,11,12,15\}$ (Left). Average occupancy on the A07 layers (Right).

the nominal $(570 \mathrm{~V})$ voltage and with the nominal Ar-CO2 (93\%-7\%) gas mixture [2]. The tracks were reconstructed according to the requirements, reported in the caption of Figure 5. The tracking efficiency was evaluated at different average levels $(\mathrm{VMM}=$ group of 64 strips, MMFE $8=$ group of $8 \mathrm{VMM}$ and $\mathrm{PCB}=$ group of $2 \mathrm{MMFE} 8)$. The efficiency at PCB-level decreases by $\sim 3 \%$ up to $60 \mathrm{mV}$ effective threshold and it is above $80 \%$ up to 60 $\mathrm{mV}$ effective threshold.

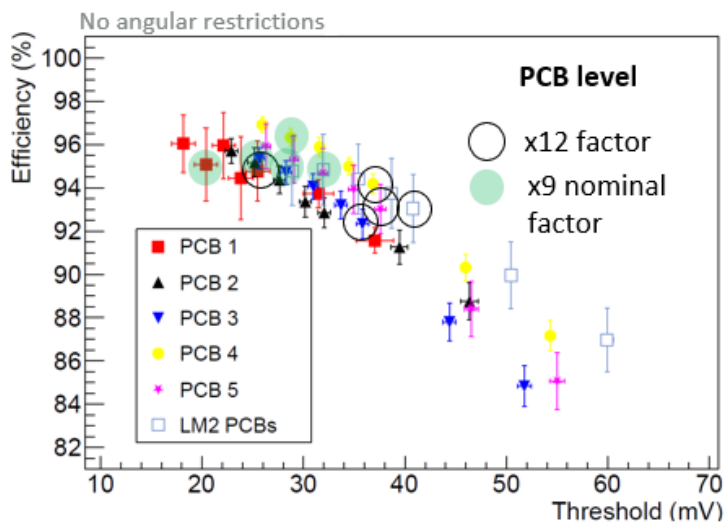

Figure 5: Efficiency at PCB-level as a function of the average effective threshold at PCB-level on the A07 layers. Full green dots are relative to the dataset with factor $\mathrm{N}=9$ and empty black dots are relative to $\mathrm{N}=12$. The tracking requirements are: no restriction on the cluster multiplicity; cut $(>1.2 \mathrm{fC})$ on the minimum charge of the strip with the maximum charge in the cluster (track projection on the readout plane) and a cut $(>0.4 \mathrm{fC}$ ) on the minimum charge of the other strips of the cluster. Together with, the requests of a maximum number of clusters in an event less than 30 and a maximum number of clusters in a layer less than 10.

\section{Conclusion}

No significant occupancy and efficiency losses were observed increasing the thresholds of $\sim 10 \mathrm{mV}$ respect to the nominal values. Specifically, the average PCB efficiency drop at the nominal voltage is around $2 \%$ from the nominal factor 9 to factor 12 . 


\section{References}

[1] ATLAS Coll., The ATLAS Experiment at the CERN Large Hadron Collider, JINST 3 (2008) S08003.

[2] ATLAS Coll., New Small Wheel Technical Design Report, (CERN-LHCC-2013-006; ATLAS-TDR-020), https://cds.cern.ch/record/1552862.

[3] Y. Giomataris et al., MICROMEGAS: a high-granularity position-sensitive gaseous detector for high particle-flux environments, NIM A376 (1996) 29.

[4] T. Alexopoulos et al., A spark-resistant bulk-micromegas chamber for high-rate applications, NIM A640 (2011) 110. 